\title{
Energy and Optical Absorption Spectra of Multiply Charged Anisotropic Quantum Boxes
}

\author{
A. Trojnar, T. MaŁachowski and A. Wóss \\ Institute of Physics, Wrocław University of Technology \\ Wybrzeże Wyspiańskiego 27, 50-370 Wrocław, Poland
}

\begin{abstract}
Using a simple model of a two-dimensional rectangular quantum box we study the effects of size and anisotropy on the energy and photoluminescence spectra of neutral and charged quantum dots. The competition of symmetries and energy/length scales of the free exciton or trion and of the confining potential is analyzed. The numerical calculations consisted of the diagonalization of the few-electron-hole Hamiltonian matrices in the full configuration-interaction basis, with the simultaneous resolution of the conserved orbital and spin quantum numbers.
\end{abstract}

PACS numbers: 73.22.-f, 78.67.Hc, 71.35.-y, 71.35.Pq

\section{Introduction}

Quantum dots [1] are nanoscale semiconductor structures in which a small, controlled number of interacting electrons and/or holes are confined to a small area/volume of designed chemical composition, size, and shape. With the typical dimensions of a few to several tens of nanometers, quantum dots are sufficiently bigger than natural atoms for the interaction effects to play a major role, instead of being completely dominated by the single-particle shell structure. On the other hand, they are still small enough for the size quantization effects to be essential. Hence, the optical properties of quantum dots are defined by the interplay of confinement and interactions.

In this paper we study the effect of size and anisotropy of the confining potential on the dynamics and recombination of confined excitonic complexes. The main problem we looked at is the reduction of symmetry when going from the isotropic Coulomb interaction responsible for the binding of an exciton or trion in the absence of an external confinement to the lower symmetry of a small, anisotropic dot. The isotropic to anisotropic transition is driven by the ratio of 
confinement and interaction length scales (dot dimension $d$ versus the Bohr radius $a_{\mathrm{B}}$ ) and energy scales (characteristic shell spacing $\hbar^{2} / 2 m d^{2}$ and the Rydberg Ry) and should in principle be observable in the size dependence of the photoluminescence $(\mathrm{PL})$ spectrum. Presented general considerations should apply for example to flat, anisotropic quantum dots made of GaAs (with $a_{\mathrm{B}} \approx 10 \mathrm{~nm}$ and $\mathrm{Ry} \approx 6 \mathrm{meV})$.

To incorporate size and anisotropy in the model we use very simple two-dimensional (2D) rectangular geometry [2,3]. Our "quantum box" has spatial dimensions $a$ and $b$, with the aspect ratio $\beta=b / a$ and the area $d^{2}=a b$ defining an average size $d$. The single particle wave functions are $\phi_{n m}=$ $a^{-1 / 2} b^{-1 / 2} \psi_{n}(x / a) \psi_{m}(y / b)$, where $\psi_{n}(X)=\sqrt{2} \sin (n \pi X)$. The energy levels are simply $\mathcal{E}_{n m}=\beta \varepsilon_{n}+\beta^{-1} \varepsilon_{m}$, where $\varepsilon_{n}=(n \hbar \pi)^{2} / 2 \mu_{\alpha} d^{2}$ and $\mu_{\mathrm{e}}$ and $\mu_{\mathrm{h}}$ are the electron and hole effective masses (we ignored hole mass anisotropy and used a fixed ratio $\mu_{\mathrm{h}} / \mu_{\mathrm{e}}=5$ ).

Two-body interaction matrix elements $\left\langle n_{1}, m_{1} ; n_{2}, m_{2}|V| n_{3}, m_{3} ; n_{4}, m_{4}\right\rangle$ were integrated using the following expansion of the Coulomb potential [4]:

$$
\frac{1}{r}=\frac{1}{\sqrt{\pi}} \int_{0}^{\infty} \frac{\mathrm{d} t}{\sqrt{t}} \mathrm{e}^{-t r^{2}}
$$

which allows us to separate the variables $x$ and $y$, and reduces the problem to a 1D numerical integration over $t$.

The single-particle, Coulomb, and excitonic energy scales are related via Ry $a_{\mathrm{B}}^{2}=\hbar^{2} / 2 \mu$ (where $\mu$ is the reduced mass, $\mu^{-1}=\mu_{\mathrm{e}}^{-1}+\mu_{\mathrm{h}}^{-1}$ ) and Ry $a_{\mathrm{B}}=e^{2} / 2$. Defining $d / a_{\mathrm{B}} \equiv \varrho$ we obtain the important scaling relation:

$$
\operatorname{Ry}: \frac{e^{2}}{2 d}: \frac{\hbar^{2}}{2 \mu d^{2}}=\varrho^{2}: \varrho: 1 \text {. }
$$

The total few-body Hamiltonian, $H=\sum_{i} \mathcal{E}_{i} c_{i}^{\dagger} c_{i}+\sum_{i j k l} V_{i j k l} c_{i}^{\dagger} c_{j}^{\dagger} c_{k} c_{l}$, written here using composite spin-orbital indexes such as $i=(n, m, \sigma)$, is diagonalized in a full configuration interaction (CI) basis including all spin configurations and all single-particle orbitals with $1 \leq n, m \leq n_{\max }$. The calculation is carried out for the exciton $(X=\mathrm{e}+\mathrm{h})$, positive and negative trions $\left(X^{ \pm}=X+\mathrm{h}\right.$ or $\left.\mathrm{e}\right)$, and for a doubly charged state $\left(X^{2-}=X+2 \mathrm{e}\right)$, as a function of $d$ and $\beta$, and for different $4 \leq n_{\max } \leq 10$ (data shown in Figs. 2 and 3 were obtained by extrapolation to $\left.n_{\max }=\infty\right)$. The resulting eigenstates conserve two total parities $\left(P_{x}\right.$ and $\left.P_{y}\right)$ and the length and projection of spin $\left(S\right.$ and $\left.S_{z}\right)$ of two or more identical particles (electrons or holes, depending on the complex). $P_{x}, P_{y}$, and $S_{z}$ are good quantum numbers of the CI basis states, and $S^{2}$ is resolved simultaneously with the Lanczos diagonalization of $H$ (by $S$-projection on each Lanczos step) [5].

\section{Results and discussion}

In Fig. 1 we show two examples of the $2 \mathrm{e}+\mathrm{h}$ energy spectrum calculated for the box size $\varrho=1$ and anisotropies $\beta=1$ and 2. Parities $P=0$ (even) and $P=1$ (odd) and the two-electron spin $S=0$ (singlet) or $S=1$ (triplet) of each 
eigenstate are indicated. Breaking of the $P_{x} \rightarrow P_{y}$ symmetry for $\beta \neq 1$ is evident. The trion ground state, whose energy we denote by $E_{X^{-}}$, is an even-even singlet, i.e., it has $\left(P_{x}, P_{y}, S\right)=(0,0,0)$ for all values of $(\varrho, \beta)$. The exciton energy $E_{X}$ (also an even-even ground state) is obtained from the analogous e $+\mathrm{h}$ spectra. The $X$ and $X^{-}$binding energies are defined as $\Delta_{X}=E_{\mathrm{e}}+E_{\mathrm{h}}-E_{X}$ (where $E_{\mathrm{e}}$ and $E_{\mathrm{h}}$ are single-electron and -hole ground states) and $\Delta_{X^{-}}=E_{X}+E_{e}-E_{X^{-}}$.

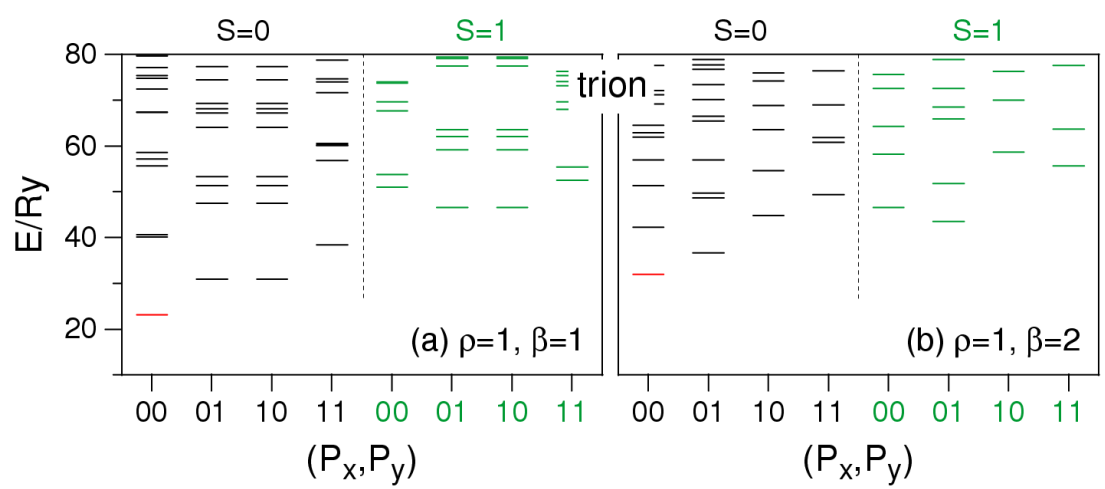

Fig. 1. Trion energy spectra in an isotropic (a) or anisotropic (b) quantum box.

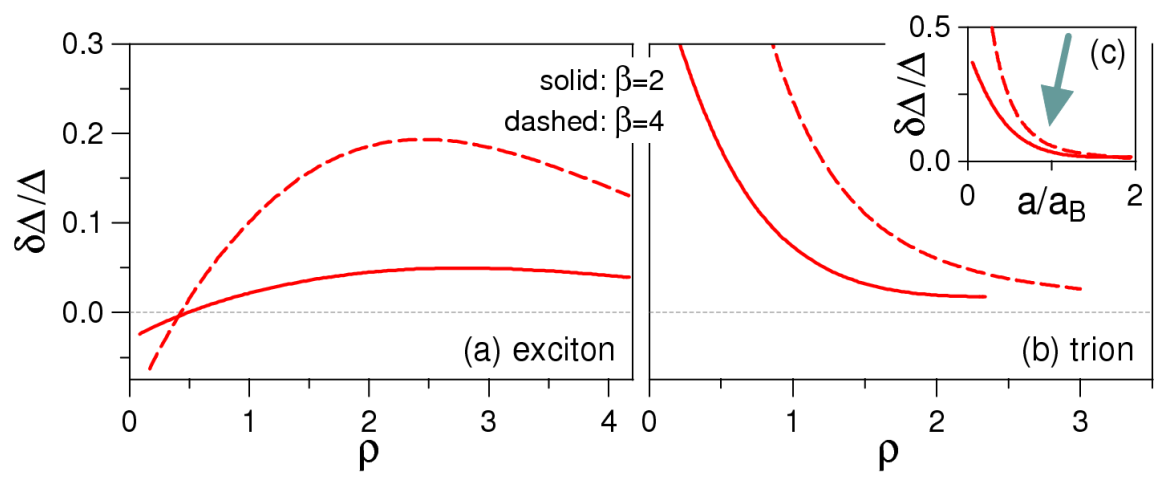

Fig. 2. Relative difference in the exciton (a) and trion (b) binding energies due to the box anisotropy $\delta \Delta / \Delta$ (see the text), plotted as a function of the box average size $\varrho$ or shorter side $a(\mathrm{c})$.

In Fig. 2 we show the relative difference in the binding energy due to box anisotropy, $\delta \Delta / \Delta=[\Delta(\beta)-\Delta(1)] / \Delta(1)$, plotted as a function of box size. The exciton and trion curves are markedly different, with the latter ones rapidly dropping to zero beyond a critical box size. It is clear from the inset (c) that the anisotropy effect depends on the shorter dimension of the box, $a$, being smaller than about the free trion radius. Thus, two size/shape regimes can be identified: (i) $a>a_{\mathrm{B}}$, with the trion moving freely inside the box and bouncing of the walls, with little 


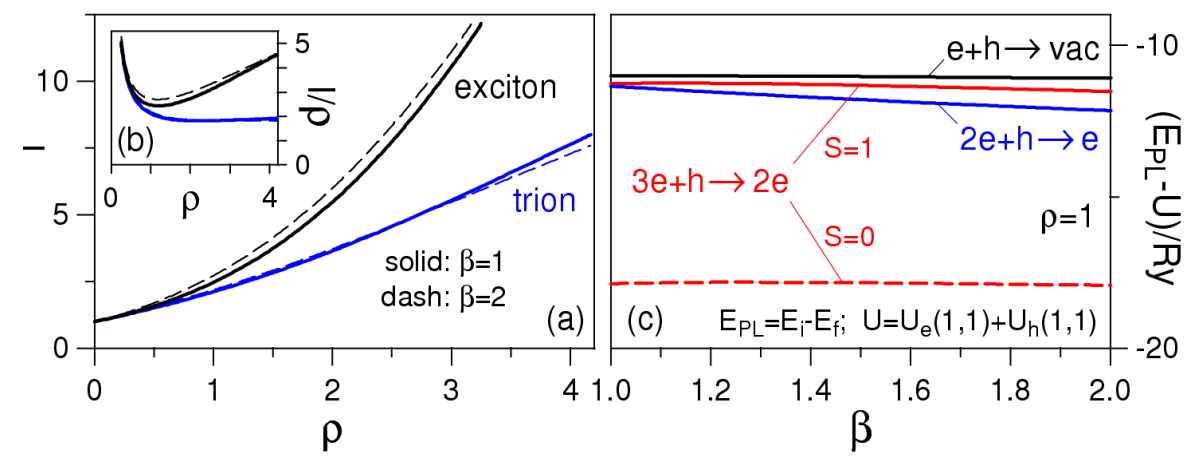

Fig. 3. (a,b) Exciton and trion PL oscillator strengths $I$ plotted as a function of the box size $\varrho$. (c) PL energy $E_{\mathrm{PL}}$ as a function of box anisotropy $\beta$.

effect of the shape or symmetry of the confinement; (ii) $a<a_{\mathrm{B}}$, with a quantized trion center of mass motion (strong confinement) and a strong anisotropy effect.

In Fig. 3 we plot the PL oscillator strengths $I$ and recombination energies $E_{\mathrm{PL}}$ (counted from the energy $U$ of a noninteracting e-h pair). The $I_{X}$ and $I_{X^{-}}$ all start at unity at $\varrho=0$, rise due to correlations in intermediate-sized boxes, and scale very differently with the box size for $\varrho \gg 1$. Anisotropy affects $I$ only for intermediate box sizes, and the effect is rather insignificant. The $E_{\mathrm{PL}}$ of Fig. 3c is also quite insensitive to $\beta$. More interesting here is the exchange splitting of the $3 \mathrm{e}+\mathrm{h}$ emission spectrum due to two possible spin states of the left-over pair of electrons.

\section{Acknowledgments}

The authors thank Pawel Hawrylak for helpful discussions and acknowledge partial support from grant N202-071-32/1513 of the Polish Ministry of Science and Higher Education.

\section{References}

[1] L. Jacak, P. Hawrylak, A. Wójs, Quantum Dots, Springer, Berlin 1998.

[2] G.W. Bryant, Phys. Rev. B 37, 8763 (1988).

[3] A. Barenco, M.A. Dupertuis, Phys. Rev. B 52, 2766 (1995).

[4] G.W. Bryant, Phys. Rev. B 31, 7812 (1985).

[5] A. Wójs, J.J. Quinn, Phys. Rev. B 75, 085318 (2007). 